\title{
Identifying and Communicating the Importance of the Variable Nature of SyS Data
}

\author{
Heather Rubino*, David Atrubin and Janet J. Hamilton
}

Epidemiology, Florida Department of Health, Tallahassee, FL, USA

\section{Objective}

This roundtable will provide a forum for national, state, and local managers of syndromic surveillance systems to discuss how they identify, monitor, and respond to changes in the nature of their data. Additionally, this session will focus on the strengths and weakness of the syndromic surveillance systems for supporting program evaluation and trend analysis. This session will also provide a forum where subject matter experts can discuss the ways in which this deep understanding of their data can be leveraged to forge and improve partnerships with academic partners.

\section{Introduction}

As syndromic surveillance systems continue to grow, new opportunities have arisen to utilize the data in new or alternative ways for which the system was not initially designed. For example, in many jurisdictions syndromic surveillance has recently become population-based, with $100 \%$ coverage of targeted emergency department encounters. This makes the data more valuable for realtime evaluation of public health and prevention programs. There has also been increasing pressure to make more data publicly available to the media, academic partners, and the general public.

\section{Keywords}

informatics; surveillance; syndromic; evaluation

\section{*Heather Rubino}

E-mail: heather.rubino@flhealth.gov 